\title{
Relationship between the chest radiograph, regional lung function studies, exercise tolerance, and clinical condition in cystic fibrosis
}

\author{
ALLAN L COATES, PAULINE BOYCE, DONALD G SHAW, SIMON GODFREY, AND \\ MARGARET MEARNS
}

Departments of Paediatrics, Hammersmith Hospital, and the Queen Elizabeth Hospital for Children, London

SUMmARY This study evaluated the accuracy of the interpretation of the chest film in delineating localised abnormalities of ventilation and perfusion, as well as the overall severity of airways obstruction, exercise tolerance, and clinical condition in children with cystic fibrosis. Radiographic findings in various regions of the chest film were compared with the functional values obtained with regional lung function tests which evaluated the arrival and disappearance of boluses of radioactive nitrogen given by inhalation and infusion. While the more severely affected areas on the chest radiograph were found to correlate with similar regions on the lung function tests, as did overall scores, errors occurred in some cases if the X-ray film alone was used as a judge of regional physiological derangement. In addition the degree of airways obstruction, the exercise tolerance on a cycle ergometer, and clinical grading, each correlated significantly with the radiographic score. We conclude that the chest radiograph is a good indicator of the overall severity of the lung disease and that it correlates well with exercise tolerance and clinical condition in cystic fibrosis.

In cystic fibrosis (CF) the interpretation of the chest radiograph is used to determine the degree and location of pulmonary abnormalities. Postural drainage is applied to those regions. The prognostic scoring systems of Taussig et al., ${ }^{1}$ and those of Shwachman and Kulczycki, ${ }^{2}$ incorporated the interpretation of the chest radiograph into their ratings. Reilly et al. ${ }^{3}$ compared a score derived from the interpretation of the chest radiograph with a score derived from pulmonary function tests and showed a good overall correlation, but they gave no information about the localisation of the disease. Alderson et al., ${ }^{4}$ using the inhalation of radioactive xenon $\left({ }^{133} \mathrm{Xe}\right)$ and monitoring its washout by a scintillation camera, found a fairly good correlation between areas of the lung with slow washout and areas with radiographically apparent disease, but not with the injection of radioactive macroaggregates. Similar results have been reported by Tam et al. ${ }^{5}$ and Gyepes et al. ${ }^{6}$ Work from this laboratory ${ }^{7}$ suggests that tests of regional lung function (RLF), using the evaluation of the arrival and subsequent clearance of the bolus of radioactive nitrogen $\left({ }^{13} \mathrm{~N}\right)$ given alternately by inhalation and intravenous injection can be used for detecting both localised and generalised pulmonary abnormalities. In view of the importance placed on the interpretation of the chest $x$-ray film both for prognosis and treatment, we sought to evaluate its sensitivity and accuracy by comparing it with other indices of pulmonary and general manifestations of CF. To do this we compared an overall radiographic score with tests of airways obstruction, exercise tolerance, and clinical condition, and with a regional radiographic score to regional abnormalities of ventilation and perfusion assessed by RLF testing.

\section{Materials and methods}

The subjects for this study were volunteers from the population of the CF clinic of the Queen Elizabeth Hospital, Hackney, London. The protocol was accepted by the Ethics Committees of the Queen Elizabeth Hospital and the Hammersmith Hospital and informed consent obtained. There were 18 children aged between 5 and 16 years in whom the diagnosis of CF had been confirmed in each by a sweat test. All showed the features typical of the disease. Their clinical condition ranged from mild to severe. Height and weight were measured using the centile charts of Tanner. $^{8}$ A clinical score was assigned to each patient by one of us (M M), using 
the scoring system of Shwachman and Kulczycki, ${ }^{2}$ modified to exclude the radiological component. Hence, each child was given a maximum of 25 points for nutritional status, physical examination, and overall wellbeing, with 75 being the maximum possible. Maximum exercise tolerance was evaluated by a progressive exercise test on a cycle ergometer. ${ }^{9}$ In this test the child pedalled continuously until exhausted, with the load being increased by a fixed increment each minute. The size of the increment $(5,10$, or 15 watts) was chosen according to the size of the child so that exhaustion would occur after between 5 and 10 minutes. The last completed work load $\left(\mathrm{W}_{\max }\right)$ was used as the measurement of exercise tolerance. The percentage of predicted values for $\mathbf{W}_{\max }$ for the child's height was calculated from the data of Godfrey. ${ }^{9}$ Maximum mid-expiratory flow (MMEF) rate was measured using a water spirometer while the patient stood, and the percentage predicted was calculated from the child's height. ${ }^{10}$ Standard inspiration chest radiographs in the posterioranterior ( $\mathrm{P}-\mathrm{A})$ and lateral position were taken on the same morning as the radioactive regional lung function study.

The technique for regional lung function studies has been described elsewhere ${ }^{7}$ and are summarised here. With the child breathing quietly while lying supine over a gamma radiation camera, a single bolus of ${ }^{13} \mathrm{~N}$ was given into a mouthpiece at functional residual capacity and inhaled into the lung with the ensuing normal tidal volume. Hence, the distribution of the ${ }^{13} \mathrm{~N}$ will be the same as the distribution of inspired air during normal breathing. Arrival and washout of the radiation was monitored continuously by the camera linked to a computer which corrected readings both for radioactive decay and background activity and then calculated activitytime curves for the study. In this study the peak counts arriving at a given region reflect the distribution of ventilation and the washout reflects the alveolar minute ventilation of this specific distribution. After the radiation from the ventilation study had washed out, a bolus of ${ }^{13} \mathrm{~N}$ dissolved in saline was given by rapid injection into the antecubital vein and the arrival and disappearance of the radioactivity in the lung from this injection were monitored in the same way. When given intravenously, the poorly soluble ${ }^{13} \mathrm{~N}$ leaves the blood to enter alveolar gas, and is removed by ventilation. The peak counts in this case reflect the distribution of the perfusion and the washout, the ventilation of the perfused regions. The total radiation dosage received depends on the weight of the child and the length of time required for the washout but it is generally between 100 and 130 mrad. In the analyses the lungs were divided into four quadrants, an upper and lower of each lung, which roughly represent equal areas as seen in the P-A projection by the camera. Ventilation and perfusion characteristics of each region were analysed and then overall lung function was evaluated by adding all regions. The index of severity used was the delay in clearance of the radiation from the lung which was quantitated as the height of the peak counts divided by the area under the washout curve $(\mathrm{H} / \mathrm{A})$ when counts were plotted against time. ${ }^{7}$ This was compared with normal data for this technique in this institution. ${ }^{71}$ While the distribution of both ventilation and perfusion changes with the supine or sitting position, the degree or location of the abnormalities does not. Hence, the supine position was chosen so as to lessen motion artefact during the 3 to 4 minutes required for the test.

In the analysis of the chest radiograph the P-A film was divided into four quadrants similar to the division of the isotope scan. The regional nature and extent of disease was evaluated by assigning up to 2 points each for the presence and severity of bronchial wall thickening, large and small areas of parenchymal radio-opacities, bronchiectasis, and areas of 'blob' consolidation as defined by Hodson and France. ${ }^{12}$ The total for the four regions gave the overall lung radiographic score. The scoring was done by one of us (D G S) without knowing the clinical status of the children or their functional measurements.

\section{Results}

The clinical and functional data of the children are shown in the Table. The age range was 5 to 16 years, but most of the children were between 11 and 13. As a group, the children were smaller than their agematched peers. Only 4 had heights greater than the 50 th centile and only one was heavier than the 50th centile. The clinical score ranged from 75 (the highest possible) to 30 . We defined those with a score of at least 60 to have a fairly mild form of the disease, and slightly more than half of the children were in this category. The correlations between the radiological score and the clinical score, the MMEF, and the $\mathrm{W}_{\max }$ are shown in Figs 1-3. Other significant correlations were the $\mathbf{W}_{\max }$ with the clinical score and MMEF $(P<0.001$ for both), the clinical score with the MMEF and the H/A perfusion with the $\mathrm{W}_{\max }(\mathrm{P}<0.05$ for both $)$.

Typical radioactive gas washout curves for the whole lung in one child both for inhalation and perfusion are shown in Fig. 4. The shaded areas represent normal values. The $\mathrm{H} / \mathrm{A}$ when the bolus of gas was by given inhalation was normal in this patient. The lower limit ( -2 standard deviations of a 
Table Age, sex, clinical score, the $M M E F$ and the $W_{\max }$ both as a percentage predicted from height, the $H / A$ for the overall perfusion and ventilation scans, and the overall radiological score

\begin{tabular}{|c|c|c|c|c|c|c|c|c|}
\hline \multirow[t]{2}{*}{ Case } & \multirow[t]{2}{*}{ Age (years) } & \multirow[t]{2}{*}{ Sex } & \multirow{2}{*}{$\begin{array}{l}\text { Clinical } \\
\text { score }\end{array}$} & \multirow{2}{*}{$\begin{array}{l}\text { MMEF \% } \\
\text { predicted }\end{array}$} & \multirow{2}{*}{$\begin{array}{l}W_{\max } \% \\
\text { predicted }\end{array}$} & \multicolumn{2}{|c|}{ Whole lung $H / A$} & \multirow{2}{*}{$\begin{array}{l}\text { Whole lung } \\
\text { radiological score }\end{array}$} \\
\hline & & & & & & Perfusion & Ventilation & \\
\hline 1 & 11 & $\mathbf{M}$ & 70 & 64 & 91 & 0.67 & $4 \cdot 93$ & 0 \\
\hline 2 & 11 & $\mathbf{M}$ & 70 & 66 & 100 & 1.45 & $2 \cdot 58$ & 1 \\
\hline 3 & 9 & $\mathbf{F}$ & 60 & 66 & 86 & $1 \cdot 59$ & 3.09 & 2 \\
\hline 4 & 5 & $\mathbf{F}$ & 75 & ND & ND & 1.49 & $2 \cdot 75$ & 2 \\
\hline 5 & 15 & $\mathbf{M}$ & 60 & 70 & 85 & $1 \cdot 14$ & $2 \cdot 30$ & 3 \\
\hline 6 & 9 & $\mathbf{M}$ & 60 & 45 & 100 & 0.88 & $1 \cdot 74$ & $4 \cdot 5$ \\
\hline 7 & 15 & $\mathbf{M}$ & 65 & 78 & 97 & 0.88 & $1 \cdot 82$ & 6 \\
\hline 8 & 15 & $\mathbf{M}$ & 70 & 44 & 103 & 0.64 & $1 \cdot 59$ & 7 \\
\hline 9 & 13 & $\mathbf{F}$ & 55 & 54 & 86 & 0.89 & $1 \cdot 50$ & 8 \\
\hline 10 & 13 & $\mathbf{M}$ & 45 & 54 & 82 & 0.69 & 2.91 & 8 \\
\hline 11 & 14 & $\mathbf{M}$ & 55 & 10 & 55 & 0.74 & $2 \cdot 76$ & 8 \\
\hline 12 & 12 & $\mathbf{F}$ & 65 & 20 & 78 & 0.60 & $2 \cdot 28$ & $8 \cdot 5$ \\
\hline 13 & 12 & $\mathbf{M}$ & 65 & 59 & 91 & 0.91 & $1 \cdot 76$ & $8 \cdot 5$ \\
\hline 14 & 10 & $\mathbf{M}$ & 50 & 20 & 57 & 0.78 & 2.01 & 10 \\
\hline 15 & 16 & $\mathbf{M}$ & 65 & 20 & 53 & 0.24 & $2 \cdot 29$ & 12 \\
\hline 16 & 11 & $\mathbf{M}$ & 35 & 12 & 50 & 0.29 & $2 \cdot 33$ & 13 \\
\hline 17 & 12 & $\mathrm{~F}$ & 30 & 15 & 49 & 0.80 & $2 \cdot 50$ & 16 \\
\hline 18 & 15 & $\mathbf{F}$ & 45 & 33 & 46 & 0.46 & $1 \cdot 33$ & 17 \\
\hline
\end{tabular}

MMEF $=$ maximum mid-expiratory flow. $\mathrm{ND}=$ not done.

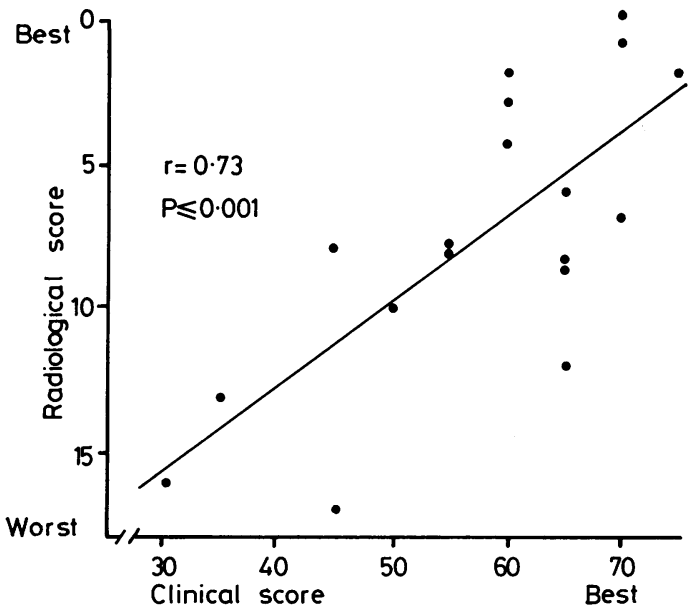

Fig. 1 Overall radiological score plotted against the clinical score.

log normal distribution) for $\mathrm{H} / \mathrm{A}$ after inhalation is $2 \cdot 23$ minutes by this technique. ${ }^{71}$ All but 7 children had values greater than this; the 7 children had values ranging from 1.33 to 2.01 minutes. ${ }^{71}$ There was no correlation between the severity of the lung disease or general condition assessed by other parameters and the $\mathrm{H} / \mathrm{A}$ of the ventilation scan.

When the radioactivity was given by infusion, the clearance from the lung was well below the lower limit of the normal range for this test (2.08 minutes) in all CF children studied. This delayed washout is shown in Fig. 4. The H/A of the perfusion washout

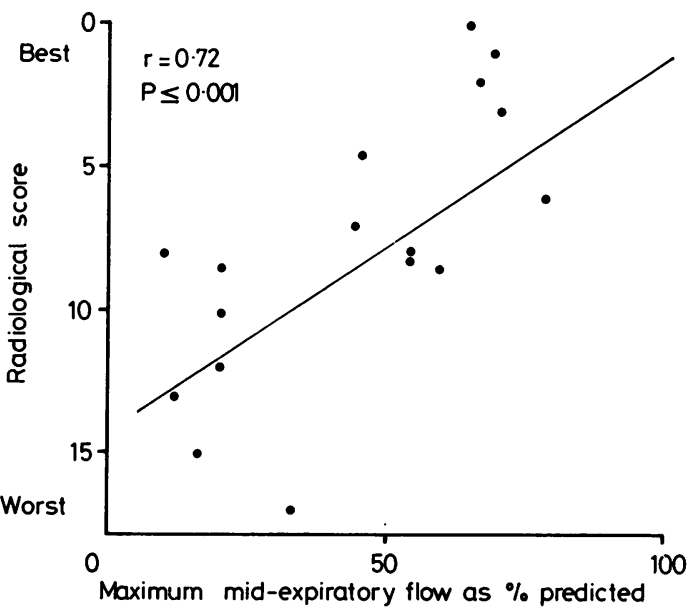

Fig. 2 Overall radiological score plotted against the $M M E F$ as a percentage of predicted.

curve correlated significantly with the other physiological evaluations, specifically the MMEF $(r=0 \cdot 64$, $P<0.01)$, and the $W_{\max }(r=0.55, P<0.05)$, but not with the clinical score. The relationship between the radiological scores and the $\mathrm{H} / \mathrm{A}$ of the perfusion studies showed a good correlation both for the lung as a whole (Fig. 5) and for individual lung regions (Fig. 6). There were a few children with various lung regions that were normal on the radiograph but abnormal by RLF testing. Conversely, there were children with lung regions with much more severe disease radiologically than was functionally 


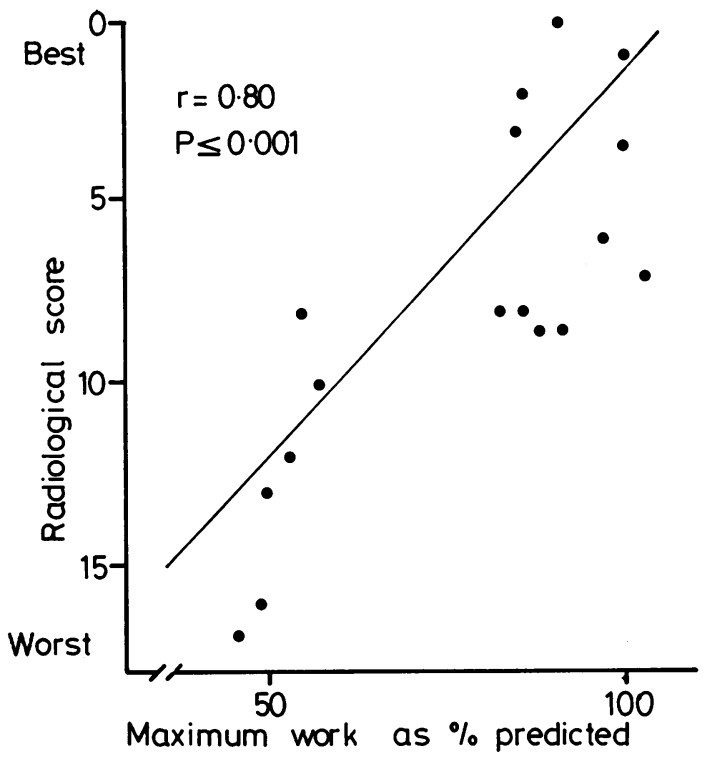

Fig. 3 Overall radiological score plotted against $W_{\max }$ as a percentage of predicted.

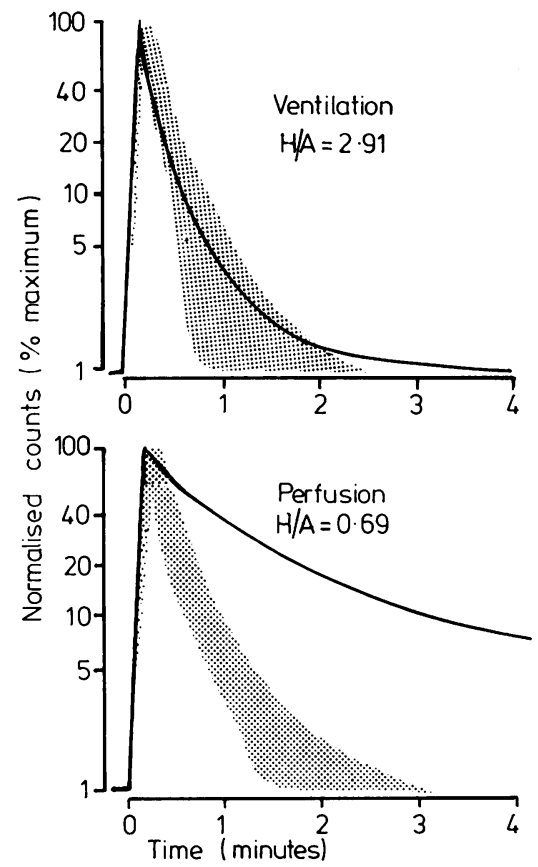

Fig. 4 A semi-logarithmic plot of radiation as a percentage of peak counts plotted against time and minutes for the ventilation scan and the perfusion scan for one of the children (Case 10). apparent by the H/A perfusion values (Fig. 6). If the radiograph showed little or no abnormality in a particular lung region but the washout of the perfusion scan of that region was abnormal, the child had a clinical score of at least 60 . In contrast, the regions with pronounced radiological abnormalities but better function all came from children with clinical scores below 30 .

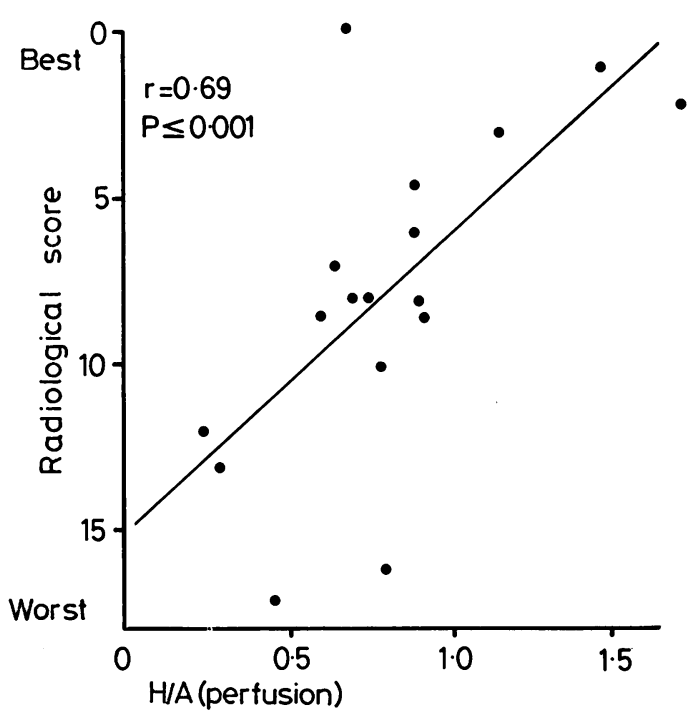

Fig. 5 Overall radiological score plotted against the height over area of the perfusion scan.

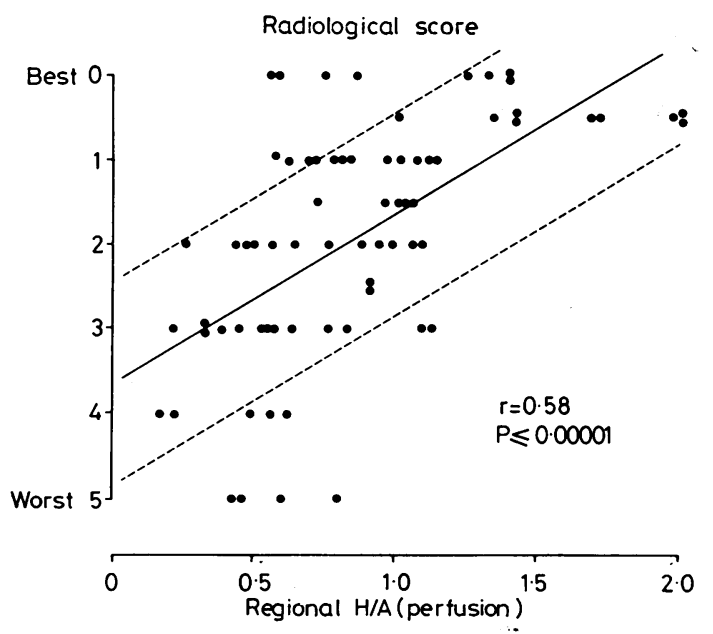

Fig. 6 Regional radiological score of each lung region plotted against the height over area of the perfusion scan for that region. The regression line (solid) and one SD (dotted) are also shown. 
When the scores of the upper regions were added up and compared with the scores of the lower regions, the upper regions were more severely affected in 10 patients using the $\mathrm{H} / \mathrm{A}$ scores and in 9 patients using the radiological scores. The lower regions were most severely affected in 5 patients using both methods, the remaining patients showing no differences.

\section{Discussion}

The results show that the overall evaluation of the chest radiograph correlates closely with other overall parameters of lung function, exercise tolerance, and general health in CF. When compared regionally, there were some discrepancies between the abnormalities assessed radiographically and those obtained by tests of RLF. These discrepancies may be due to scattered small regions of well perfused but poorly ventilated parts of the lung lying distal to obstructed small airways. Abnormalities in these small airways have been shown to be one of the early findings in cystic fibrosis. ${ }^{13}$ These early changes would not necessarily show up on the chest $x$-ray film, but would cause pronounced abnormalities in the isotope scan since ${ }^{13} \mathrm{~N}$ delivered to an obstructed alveolus by perfusion would be very slow to wash out by ventilation. However, bronchial wall thickening, bronchiectasis, or atelectasis, while very prominent on the chest film due to the associated increased tissue density, would not necessarily result in gas-trapping and would therefore not be seen on the scan. The lack of a significant correlation between the perfusion scan and the clinical score is due to the great variability of the H/A in children with high clinical scores, reflecting the sensitivity of the scan in detecting evidence of impairment before it is clinically or radiologically evident.

One of the assumptions made in our analysis is that the bolus of ${ }^{13} \mathrm{~N}$ arrives instantaneously. Should the 'bolus' arrive within a period of a few seconds, some of the isotope would be washed out of the fast areas of the lung before the entire bolus had arrived. The net effect of this would be to lower the peak counts. This would result in a slight exaggeration of the abnormalities provided that both fast and slow regions existed, since only the fast areas would be cleared during the arrival of the 'bolus'. If there was little inhomogeneity as in normal lungs where all regions are fast, then both height and area would be reduced together with little effect on the ratio.

The fact that the ventilation scan failed to show differences in severity of pulmonary derangement must be because all children, regardless of the severity of disease, had some well ventilated parts of their lungs, and almost all of the inhaled bolus of ${ }^{13} \mathrm{~N}$ went there, bypassing the more obstructed regions which would be the areas that would be radiologically more apparent. Presumably, with progression of their disease, the proportion of lung that receives any significant amount of ventilation becomes smaller and smaller. When looked at regionally, variations of the $\mathrm{H} / \mathrm{A}$ of the ventilation scan tended to be parallel with those of the perfusion scan, but the latter was much more sensitive in distinguishing the various degrees of abnormalities. This might explain the difference between our results and those of other investigators, ${ }^{4-5}$ who used a steady state ${ }^{133} \mathrm{Xe}$ inhalation technique. They did not give the radioactive gas intravenously, but instead used intravenously-administered labelled macroaggregates to evaluate pulmonary blood flow. They were able to show some abnormalities in the steady state ventilation scan which correlated with the chest radiograph, but they were unable to demonstrate the gross mismatching of ventilation and perfusion which we found after giving ${ }^{13} \mathrm{~N}$ intravenously. While macroaggregates will demonstrate the distribution of pulmonary blood flow, only a label which is delivered to the alveoli in proportion to their blood flow and then cleared at a rate proportional to their ventilation will demonstrate ventilation perfusion abnormalities due to the presence of well-perfused, poorly-ventilated alveoli.

The functional tests which we compared with the RLF tests are available and suitable for use in children. The progressive exercise test was chosen for its reproducibility and simplicity. It requires only a few minutes of intense energy compared with the much longer and more rigorous test for the determination of maximum oxygen consumption. The MMEF was preferred to other tests of airways obstruction because it is probably one of the most widely used tests of airways obstruction, and requires only the use of a simple water spirometer. Although the more sophisticated test of comparing flow volume curves obtained while breathing a mixture of helium and oxygen has been shown to be more sensitive in detecting early abnormalities in CF than tests while breathing air, there still exists a good correlation between these tests and the MMEF.13

In conclusion, we have demonstrated that the interpretation of the chest radiograph correlates well with other indices of health in children with CF. It also compares favourably with the more sophisticated tests of RLF. We believe that in most clinical situations there is little additional information to be gained from RLF tests that is not available from the interpretation of the chest radiograph and pulmonary function test. However, if RLF tests are to be done, we stress the usefulness of the intravenous administration of an insoluble gas in order to 
demonstrate ventilation-perfusion abnormalities at the alveolar level.

\section{A L C was a Fellow of the Canadian Medical Research Council, and P B was supported by the British Cystic Fibrosis Foundation.}

\section{References}

1 Taussig L M, Kattwinkel J, Friedwald W T, di Sant' Agnese P A. A new prognostic score and clinical evaluation system for cystic fibrosis. J Pediatr 1973; 82: 380-90.

? Shwachman H, Kulczycki L L. Long study of one hundred and five patients with cystic fibrosis. Am J Dis Child 1958; 96: 6-15.

3 Reilly B J, Featherby E A, Weng T R, Crozier D N, Duie A, Levison $\mathbf{H}$. The correlation of radiological changes with pulmonary function in cystic fibrosis. Radiology 1971;98: 281-5.

4 Alderson P O, Secker-Walker R H, Strominger D B, McAlister W H, Hill R L, Markham J. Quantitative assessment of regional ventilation and perfusion in children with cystic fibrosis. Radiology $1974 ; 111$ : 151-5.

5 Tam C H, Mansell A L, Levison H, Reilly B J, Aspin N. Dynamic regional lung function studies in patients with asthma and cystic fibrosis. Am Rev Respir Dis 1973; 108 . 283-93.

6 Gyepes M T, Bennett L R, Hassakis P C. Regional pulmonary blood flow in cystic fibrosis. $A J R$ 1969; 106: 567-75.

7 Ronchetti R, Stocks J, Freedman N, Glass H, Godfrey S. Clinical application of regional lung function studies in infants and small children using ${ }^{13}$ N. Arch Dis Child 1975; 50: 595-603.

8 Tanner J M. Modern trends in paediatrics. London: Butterworth, 1958.

9 Godfrey S. Exercise testing in children. Philadelphia: Saunders, 1974.

10 Polgar G, Promadhat V. Pulmonary function testing in children. Philadelphia: Saunders, 1971: 187.

11 Godfrey S, Mackenzie S. The place of radioisotopic lung function studies in paediatrics. Arch Dis Child 1978; 52: 859-64.

12 Hodson C J, France N E. Pulmonary changes in cystic fibrosis of the pancreas: a radio-pathological study. Clin Radiol 1962; 13: 54-61.

13 Fox W W, Bureau M A, Taussig L M, Martin R R, Beaudry $\mathrm{P}$ H. Helium flow volume curves in the detection of early small airway disease. Pediatrics 1974 ; 54: 293-9.

Correspondence to Dr A L Coates, Suite D-380, Montreal Children's Hospital, 2300 Tupper Street, Montreal, Quebec H3H 1P3, Canada.

Received 27 November 1979 